\title{
Three-dimensional natural convection in a porous cavity by the boundary element method
}

\author{
R. Jecl ${ }^{1}$, J. Kramer ${ }^{1}$, J. Ravnik ${ }^{2}$ \& L. Š Skerget ${ }^{2}$ \\ ${ }^{1}$ Faculty of Civil Engineering, University of Maribor, Slovenia \\ ${ }^{2}$ Faculty of Mechanical Engineering, University of Maribor, Slovenia
}

\begin{abstract}
A thee-dimensional numerical simulation of convective flow in porous media using an algorithm based on a combination of a single domain and a subdomain boundary element method (BEM) is presented. The fluid flow in porous media is modeled with the modified Navier-Stokes equations (Brinkman-extended Darcy formulation with inertial term included) coupled with the energy and species equations using the Boussinesq approximation. The velocity-vorticity formulation is adopted to solve the governing set of equations, which results in decoupling of the computational scheme into the kinematic and kinetic computational parts. The boundary vorticity values are calculated by a single domain BEM solution of the kinematics equation, while the subdomain BEM is used to solve the vorticity, energy and species transport equations. Computations are performed for various governing parameters (Rayleigh number, Darcy number, Lewis number, buoyancy coefficient) and, simulation results are compared to the results of some published studies. Heat and mass flux through the cavity and flow fields are analyzed, focusing on the 3D nature of the phenomena.

Keywords: boundary element method, porous medium, three-dimensional natural convection, Brinkman-extended Darcy formulation.
\end{abstract}

\section{Introduction}

Problems of convective flow in saturated porous media can be found in a wide variety of engineering and natural applications, e.g. building thermal insulation, extraction of geothermal energy, heat exchangers, contaminant transport through water saturated soil. Most of the published studies, which are dealing with buoyancy induced flows in porous enclosures, are limited on the cases of two 
dimensional geometries where the fluid flow in porous media is modeled with the general linear law of permeability (Darcy law). Later, the fluid flow model was extended with the Forchheimer term which represents additional non-linear inertia term due to increasing velocity. The most commonly used extension of Darcy law is with the addition of the terms covering the influence of inertia effects analogous to the Navier-Stokes equations and Brinkman viscous term, which enables to satisfy the no-slip boundary condition on impermeable surfaces that bound the porous media. The so called Brinkman model is also used in the present study.

Studies of buoyancy induced flows in three-dimensional porous configurations are rare and primary confined to conditions of heating from below e.g. [1-3], only few can be found considering 3D enclosure heated from the side e.g. [46]. Furthermore, some 3D results of double diffusive natural convection, with combined actions of thermal and solutal buoyancy forces in porous enclosures can be found in [7] and [8].

Numerous numerical methods have been proposed to simulate the natural convection phenomena in porous media. In the present study, the velocity-vorticity formulation is employed to the macroscopic Navier-Stokes equations, written for the porous media, which are coupled with the energy and species equations. The unknown field functions are velocity, vorticity, temperature and concentration. The Boundary Element Method (BEM) is employed to find the solution. The proposed algorithm is based on the pure fluid [9] and nanofluid [10] simulation codes. The boundary vorticity values are calculated using the single domain BEM, while all other flow fields are solved by subdomain BEM. The vorticity, energy and species transport equations are solved using a domain decomposition approach [11].

\section{Mathematical formulation}

\subsection{Geometry of the problem}

The geometry under consideration is a cube enclosure, shown in Fig. 1, filled with porous media, which is fully saturated with incompressible fluid. Left and right vertical walls are imposed to different temperature and concentration values, where $T_{1}>T_{2}$ and $C_{1}>C_{2}$, while the remaining boundaries are adiabatic and impermeable.

Due to subjected temperature and concentration differences on two vertical walls, the natural convection phenomena in the enclosure will occur. The density of the heated fluid next to the hot wall decreases and buoyancy will carry it upwards. On the other hand, fluid along the cold wall will be colder and denser and it will travel downwards. Due to applied concentration differences on the walls additional concentration buoyancy forces are induced, which cause additional movement of the fluid. Both induced buoyancy forces can aid or oppose each other, which also influences the strength of the convective motion of the fluid. The cases when solute is transported due to induced temperature gradient (Soret effect) or heat is transferred due to concentration gradient (Dufour effect) are neglected in the present study. 


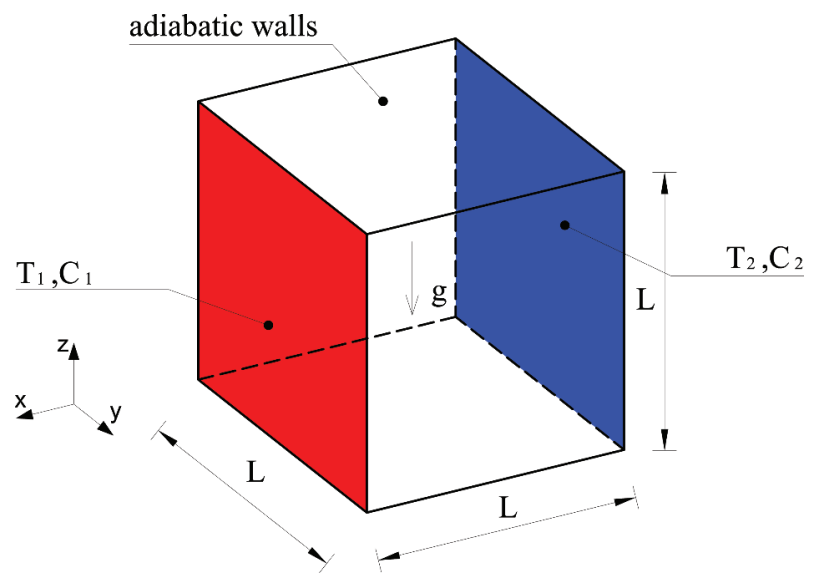

Figure 1: Geometry with boundary conditions for convection with horizontal temperature and vertical solutal gradient.

\subsection{Governing equations}

The governing equations for the problem of double-diffusive natural convection in porous media are given in terms of conservation laws for mass, momentum, energy and species. They are obtained from classical Navier-Stokes equations for the pure fluid flow which are generally written at the microscopic level. By volume averaging over suitable representative elementary volume (REV) and considering the fact that only a part of this volume, expressed with the porosity $\phi$, is available for fluid flow, macroscopic or volume averaged Navier-Stokes equations can be derived. The averaging procedure is given in detail in [12].

The REV has to be determined in a way that, irrespective of its position in porous media, it always contain both solid and fluid phases. In the model development following assumptions are adopted: the fluid flow is steady and laminar, the solid phase is homogeneous, isotropic and non-deformable, the fluid is incompressible Newtonian and in thermal equilibrium with the solid phase. The porosity and permeability of porous medium are constant while the density of the fluid depends only on temperature and concentration variations and is described with the Oberbeck Boussinesq approximation as:

$$
\rho=\rho_{0}\left(1-\beta_{T}\left(T-T_{0}\right)-\beta_{C}\left(C-C_{0}\right)\right) .
$$

In above expression $\rho$ is density of the fluid, $\beta_{T}$ is the volumetric thermal expansion coefficient, $T$ is temperature, $\beta_{C}$ is the volumetric expansion coefficient due to chemical species and $C$ is concentration. The subscript 0 refers to a reference state.

Further assumption is that no internal energy sources are present in the fluid saturated porous media. The irreversible viscous dissipation is also neglected, while no high velocity flow of highly viscous fluid is considered in the present 
study. The solid phase of porous medium is assumed to be in thermal equilibrium with the saturating fluid.

The macroscopic conservation equations can now be written as:

- continuity equation

$$
\vec{\nabla} \cdot \vec{v}=0
$$

- momentum equation

$$
\frac{1}{\phi} \frac{\partial \vec{v}}{\partial t}+\frac{1}{\phi^{2}}(\vec{v} \cdot \vec{\nabla}) \vec{v}=-\left(\beta_{T}\left(T-T_{0}\right)+\beta_{C}\left(C-C_{0}\right)\right) \vec{g}-\frac{1}{\rho} \vec{\nabla} p+\frac{1}{\phi} \nu \nabla^{2} \vec{v}-\frac{\nu}{K} \vec{v},
$$

- energy equation

$$
\sigma \frac{\partial T}{\partial t}+(\vec{v} \cdot \vec{\nabla}) T=\lambda_{e} / c_{f} \nabla^{2} T
$$

- species equation

$$
\phi \frac{\partial C}{\partial t}+(\vec{v} \cdot \vec{\nabla}) C=D \nabla^{2} C
$$

The parameters used above are: $\vec{v}$ volume averaged velocity, $\phi$ porosity, $t$ time, $\rho$ density, $\nu$ kinematic viscosity, $p$ pressure, $\vec{g}$ gravity vector, $K$ permeability. In the energy equation $\sigma$ represents the heat capacity ratio $\sigma=\left(\phi c_{f}+(1-\phi) c_{s}\right) / c_{f}$, where $c_{f}=\left(\rho c_{p}\right)_{f}$ and $c_{s}=\left(\rho c_{p}\right)_{s}$ are heat capacities for fluid and solid phases respectively. $\lambda_{e}$ is the effective thermal conductivity of the fluid saturated porous media given as $\lambda_{e}=\phi \lambda_{f}+(1-\phi) \lambda_{s}$, where $\lambda_{f}$ and $\lambda_{s}$ are thermal conductivities for fluid and solid phases respectively. In the species equation $C$ is concentration, and $D$ mass diffusivity.

The momentum equation (3) is also known as the Darcy-Brinkman equation, with two viscous terms e.g. Brinkman viscous term (third on the r.h.s) and Darcy viscous term (fourth on the r.h.s.). The Brinkman viscous term is analogous to the Laplacian term in the classical Navier-Stokes equations for pure fluid flow. It expresses the viscous resistance or viscous drag force exerted by the solid phase on the flowing fluid at their contact surfaces. With the Brinkam term the non-slip boundary condition on a surface which bounds porous media is satisfied [13].

\subsection{Velocity-vorticity formulation}

The velocity-vorticity formulation of above given modified Navier-Stokes equations is derived by taking the curl of the mass conservation law (2) and of the Brinkman momentum equation (3). The vorticity is defined as the curl of the velocity field $\vec{\omega}=\vec{\nabla} \times \vec{v}$ and is solenoidal by the definition, $\vec{\nabla} \cdot \vec{\omega}=0$. As a consequence of the transformation, the computational scheme is partitioned into its kinematic and kinetic parts [14]. The kinematics is given with the elliptic velocity vector equation:

$$
\nabla^{2} \vec{v}+\vec{\nabla} \times \vec{\omega}=0
$$


furthermore, the kinetic part is governed by the vorticity transport equation:

$$
\begin{aligned}
\phi \frac{\partial \vec{\omega}}{\partial t}+(\vec{v} \cdot \vec{\nabla}) \vec{\omega}= & (\vec{\omega} \cdot \vec{\nabla}) \vec{v}-\phi^{2} \vec{\nabla} \times\left(\beta_{T}\left(T-T_{0}\right)+\beta_{C}\left(C-C_{0}\right)\right) \vec{g}+ \\
& +\nu \phi \nabla^{2} \vec{\omega}-\frac{\nu \phi^{2}}{K} \vec{\omega} .
\end{aligned}
$$

\subsection{Non-dimensional equations}

Before all equations will be rewritten in the non-dimensional form, in the vorticity, energy and species equations the modified vorticity, temperature and concentration time steps are introduced as $t_{\omega}=t / \phi, t_{T}=t / \sigma$ and $t_{C}=t / \phi$. These are necessary mathematical steps allowing to use the numerical scheme presented in following chapter.

Non-dimensional form of the governing equations is adopted, using following dimensionless variables:

$$
\begin{aligned}
& \vec{v} \rightarrow \frac{\vec{v}}{v_{0}}, \vec{r} \rightarrow \frac{\vec{r}}{L}, \vec{\omega} \rightarrow \frac{\vec{\omega} L}{v_{0}}, t \rightarrow \frac{v_{0} t}{L}, t_{\omega} \rightarrow \frac{v_{0} t_{\omega}}{L}, t_{T} \rightarrow \frac{v_{0} t_{T}}{L}, \\
& t_{C} \rightarrow \frac{v_{0} t_{C}}{L}, T \rightarrow \frac{T-T_{0}}{\Delta T}, C \rightarrow \frac{C-C_{0}}{\Delta C}, \vec{g} \rightarrow \frac{\vec{g}}{g_{0}},
\end{aligned}
$$

where $v_{0}$ is characteristic velocity, $\vec{r}$ position vector and $L$ characteristic length. Furthermore, $T_{0}$ and $C_{0}$ are characteristic temperature and concentration, $\Delta T$ and $\Delta C$ are characteristic temperature and concentration differences and $g_{0}=$ $9,81 \mathrm{~m} / \mathrm{s}^{2}$ gravity acceleration. Characteristic velocity is given with the expression $v_{0}=\lambda_{f} /\left(c_{f} L\right)$, where $\lambda_{f}$ is thermal conductivity of the fluid. This definition is common when considering buoyant flow simulations.

The macroscopic non-dimensional vorticity equation can now be written as:

$$
\begin{aligned}
\frac{\partial \vec{\omega}}{\partial t_{\omega}}+(\vec{v} \cdot \vec{\nabla}) \vec{\omega}= & (\vec{\omega} \cdot \vec{\nabla}) \vec{v}-\operatorname{Pr} R a_{T} \phi^{2} \vec{\nabla} \times(T+N C) \vec{g}+ \\
& +\operatorname{Pr} \phi \nabla^{2} \vec{\omega}-\frac{\operatorname{Pr}}{D a} \phi^{2} \vec{\omega}
\end{aligned}
$$

with non-dimensional governing parameters defined as:

- $\operatorname{Pr}$, Prandtl number:

$$
\operatorname{Pr}=\frac{\nu}{\alpha}
$$

where $\nu$ is kinematic viscosity and $\alpha$ thermal diffusivity given as $\alpha=$ $\lambda_{f} / c_{f}$.

- $R a_{T}$, thermal fluid Rayleigh number:

$$
R a_{T}=\frac{g \beta_{T} \Delta T L^{3}}{\nu \alpha}
$$


- $R a_{P}$, porous Rayleigh number:

$$
R a_{P}=R a_{T} D a=\frac{g \beta_{T} \Delta T L K}{\nu \alpha},
$$

where

- $D a$ is a Darcy number:

$$
D a=\frac{K}{L^{2}},
$$

and $K$ is permeability of porous media,

- $N$, buoyancy coefficient:

$$
N=\frac{R a_{S}}{R a_{T}}
$$

where

- $R a_{S}$ is solutal Rayleigh number:

$$
R a_{S}=\frac{g \beta_{C} \Delta C L^{3}}{\nu \alpha}
$$

The energy conservation equation in the non-dimensional form can be written as:

$$
\frac{\partial T}{\partial t_{T}}+(\vec{v} \cdot \vec{\nabla}) T=\frac{\lambda_{e}}{\lambda_{f}} \nabla^{2} T
$$

and finally the species conservation equation in non-dimensional form reads:

$$
\frac{\partial C}{\partial t_{C}}+(\vec{\nabla} \cdot \vec{v})=L e \nabla^{2} C
$$

where $L e$ is Lewis number, given with expression:

$$
L e=\frac{\alpha}{D} .
$$

\section{Numerical method}

Considering a domain $\Omega$ with a boundary $\Gamma$ a fundamental solution of the Laplace equation and the Gauss and Greens theorems are used to write the integral kinematics equation without derivatives of the velocity or vorticity fields [15]:

$$
c(\xi) \vec{v}(\xi)+\int_{\Gamma} \vec{v}(\vec{n} \cdot \vec{\nabla}) u^{\star} d \Gamma=\int_{\Gamma} \vec{v} \times(\vec{n} \times \vec{\nabla}) u^{\star} d \Gamma+\int_{\Omega}\left(\vec{\omega} \times \vec{\nabla} u^{\star}\right) d \Omega .
$$

Here $\vec{\xi}$ is the source or collocation point, $\vec{n}$ is a vector normal to the boundary, pointing out of the domain and $u^{\star}$ is the fundamental solution: $u^{\star}=1 / 4 \pi|\vec{\xi}-\vec{r}|$. In order to have a nonsingular system of equations for solving for boundary values of vorticity, a tangential form of equation (19) is used. It is obtained by a cross 
product with a unit normal, yielding:

$$
\begin{aligned}
& c(\vec{\xi}) \vec{n}(\vec{\xi}) \times \vec{v}(\vec{\xi})+\vec{n}(\vec{\xi}) \times \int_{\Gamma} \vec{v} \vec{\nabla} u^{\star} \cdot \vec{n} d \Gamma \\
= & \vec{n}(\vec{\xi}) \times \int_{\Gamma} \vec{v} \times(\vec{n} \times \vec{\nabla}) u^{\star} d \Gamma+\vec{n}(\vec{\xi}) \times \int_{\Omega}\left(\vec{\omega} \times \vec{\nabla} u^{\star}\right) d \Omega .
\end{aligned}
$$

The same fundamental solution and a standard BEM derivation [16] is used to write the integral forms of the vorticity transport equation (9), the energy equation (16) and species equation (17):

$$
\begin{gathered}
c(\vec{\xi}) \omega_{j}(\vec{\xi})+\int_{\Gamma} \omega_{j} \vec{\nabla} u^{*} \cdot \vec{n} d \Gamma=\int_{\Gamma} u^{*} q_{j} d \Gamma+ \\
+\frac{1}{\operatorname{Pr}} \frac{1}{\phi}\left(\int_{\Gamma} \vec{n} \cdot\left\{u^{*}\left(\vec{v} \omega_{j}-\vec{\omega} v_{j}\right)\right\} d \Gamma-\int_{\Omega}\left(\vec{v} \omega_{j}-\vec{\omega} v_{j}\right) \cdot \vec{\nabla} u^{*} d \Omega\right)- \\
-\operatorname{Ra_{f}\phi } \int_{\Gamma}\left(u^{*} T \vec{g} \times \vec{n}\right)_{j} d \Gamma-R a_{f} \phi \int_{\Omega}\left((T+N \cdot C) \vec{\nabla} \times u^{*} \vec{g}\right)_{j} d \Omega+ \\
+\frac{1}{D a} \phi \int_{\Omega} \omega_{j} u^{*} d \Omega, \\
c(\vec{\xi}) T(\vec{\xi})+\int_{\Gamma} T \vec{\nabla} u^{*} \cdot \vec{n} d \Gamma=\int_{\Gamma} u^{*} q_{T} d \Gamma+ \\
\quad+\frac{\lambda_{f}}{\lambda_{e}}\left(\int_{\Gamma} \vec{n} \cdot\left\{u^{*}(\vec{v} T)\right\} d \Gamma-\int_{\Omega}(\vec{v} T) \cdot \vec{\nabla} u^{*} d \Omega\right) . \\
c(\vec{\xi}) C(\vec{\xi})+\int_{\Gamma} C \vec{\nabla} u^{*} \cdot \vec{n} d \Gamma=\int_{\Gamma} u^{*} q_{C} d \Gamma+ \\
+D\left(\int_{\Gamma} \vec{n} \cdot\left\{u^{*}(\vec{v} C)\right\} d \Gamma-\int_{\Omega}(\vec{v} C) \cdot \vec{\nabla} u^{*} d \Omega\right) .
\end{gathered}
$$

Here, $\omega_{j}$ is a vorticity component, $q_{j}$ is a component of vorticity flux, $q_{T}$ is the heat flux and $q_{C}$ is the species flux. In the present study only steady flow fields will be considered, thus the time derivative terms $\partial \omega / \partial t_{\omega}, \partial T / \partial t_{T}$ and $\partial C / \partial t_{C}$ are omitted. A combination of subdomain BEM and single domain BEM for the solution of the governing equations will be applied. The Dirichlet and/or Neumann boundary conditions for velocity, temperature and concentration are given. They are used to obtain solutions of the kinematics equation (19) for domain velocity values, energy equation (22) for domain temperature values and species equation (23) for domain concentration values. The boundary conditions for vorticity, which are needed to solve the vorticity transport equation (21), are unknown. The single domain BEM on the tangential form of the integral kinematics equation (20) will be used to obtain the unknown boundary vorticity values.

In the subdomain BEM method, which is used to solve equations (19), (21) and (22) a mesh of the entire domain $\Omega$ is made, each mesh element is named as a subdomain. Equations are written for all source points on each of the subdomains. 
In order to obtain a discrete version of integral equations shape functions are used to interpolate field functions and flux across the boundary and inside of the subdomain. In this work hexahedral subdomains with 27 nodes are used, which enable continuous quadratic interpolation of field functions. The boundary of each hexahedron consists of 6 boundary elements. On each boundary element the flux is interpolated using discontinuous linear interpolation scheme with 4 nodes. By using discontinuous interpolation, flux definition problems in corners and edges could be avoided. Between subdomains the functions and their fluxes are assumed to be continuous. The resulting linear systems of equations are over-determined and sparse. They are solved in a least-squares manner. Discretization procedure for the single domain BEM, which is used to solve equation (20), is analogous, with a distinction that source points are set into all nodes on the boundary of the entire domain. The resulting linear system of equations is full. It is solved by the LU decomposition method. This algorithm has been proposed for $3 D$ fluid flow and heat transfer by Ravnik et al. [9]. In present work, the algorithm for simulations in porous media was expanded. The kinematics equation requires no changes, while porous parameters had to be introduced in vorticity transport and energy equations. The Darcy term in the vorticity transport equation, which is not present in the pure fluid case, is linearly proportional to the unknown vorticity, thus it was included into the system matrix.

\section{Results and discussion}

Natural convection phenomena in fluid saturated porous medium is expected to depend on a number of parameters such as porosity, thermal conductivity and heat capacity of fluid and solid phases, viscosity of the fluid phase etc.. The wall heat and species fluxes are calculated for different values of porous Rayleigh number $\left(R a_{P}=50,100,200,500\right.$ and 1000), Darcy numbers $\left(10^{-6} \leq D a \leq 10^{-1}\right)$, Lewis number $L e=10$ and buoyancy coefficient $N=1$, which can be expressed in terms of average Nusselt and Sherwood numbers given as:

$$
N u=\int_{\Gamma} \vec{\nabla} T \cdot \vec{n} d \Gamma, \quad S h=\int_{\Gamma} \vec{\nabla} C \cdot \vec{n} d \Gamma .
$$

$\Gamma$ is the surface through which the heat and species fluxes are calculated and $\vec{n}$ is the unit normal to this surface.

The calculations were performed on a nonuniform mesh with $20 \times 8 \times 20$ subdomains and 28577 nodes. Subdomains are concentrated towards the hot and the cold walls. The convergence criteria for all field functions was $10^{-5}$, underrelaxation of vorticity, temperature and concentration values ranging from 0.1 to 0.01 was used.

Table 1 presents Nusselt number values for the cubic enclosure for $\operatorname{Pr}=0.71$, $\phi=0.8, R a_{P}=1000$ and different values of $D a$. The results are compared to the study of authors R. V. Sharma and R. P. Sharma [5], where the 3D natural convection in a porous box is considered, and the fluid flow is modeled with use of the Darcy-Brinkman-Forchheimer model. Very good agreement between 
Table 1: Nusselt number values for the $3 D$ natural convection in a cube for $R a_{P}=1000, \phi=0.8$ and different values of Darcy number. The results are compared to study of Sharma and Sharma [5].

\begin{tabular}{lccccccc}
\hline$R a_{P} / D a$ & & $10^{-1}$ & $10^{-2}$ & $10^{-3}$ & $10^{-4}$ & $10^{-5}$ & $10^{-6}$ \\
\hline \multirow{2}{*}{1000} & present & 1.855 & 3.770 & 6.922 & 10.558 & 13.242 & 14.568 \\
& {$[5]$} & - & 3.99 & 6.95 & 10.14 & 12.78 & 13.72 \\
\hline
\end{tabular}

the results can be observed for the case of high Darcy number values $(\mathrm{Da}=$ $\left.10^{-4}-10^{-2}\right)$. Slight differences occur in a case of very low Darcy numbers ( $D a=10^{-5}$ and $10^{-6}$ ). In this case the effect of Forchheimer term, which is not included in the model of the present study, becomes significant and influences the overall heat transfer resulting in lower values of Nusselt numbers [5].

In addition, Nusselt number values for natural convection for $\operatorname{Pr}=0.71$, $\phi=0.8, R a_{P}=50,100,200,500$ and 1000 and $10^{-6} \leq D a \leq 10^{-1}$ are presented graphically in Fig. 2.

It can be observed, that the Nusselt number is increasing with decrease of $D a$ and increase of $R a_{P}$. The influence of Darcy number is more pronounced

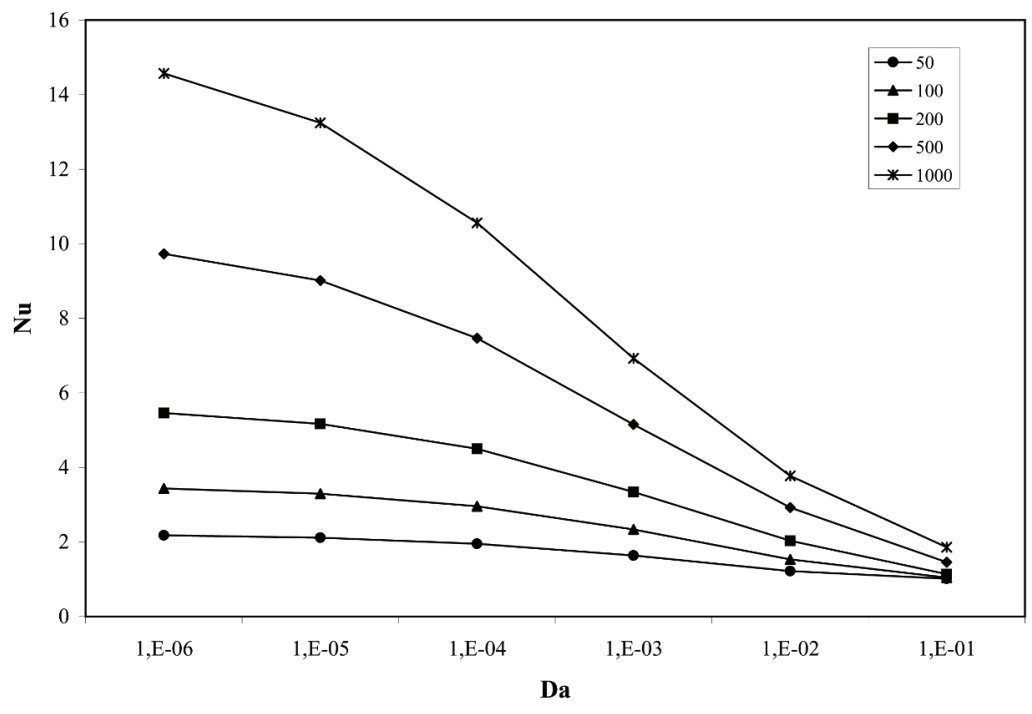

Figure 2: Dependence of the Nusselt number on the Darcy number for different values of porous Rayleigh number. 
Table 2: Nusselt and Sherwood number values for the $3 D$ natural convection in a cube for $R a_{P}=100, L e=0, N=0$ and $L e=10, N=1, \phi=0.8$ and different values of Darcy number.

\begin{tabular}{lllllllc}
\hline$R a_{P} / D a$ & & $10^{-1}$ & $10^{-2}$ & $10^{-3}$ & $10^{-4}$ & $10^{-5}$ & $10^{-6}$ \\
\hline$L e=0, N=0$ & $N u$ & 1.039 & 1.533 & 2.331 & 2.957 & 3.295 & 3.431 \\
$L e=10, N=1$ & $N u$ & 1.086 & 1.687 & 2.529 & 3.164 & 3.595 & 3.788 \\
& $S h$ & 2.842 & 5.624 & 9.749 & 14.714 & 19.107 & 20.869 \\
\hline
\end{tabular}

at higher values of porous Rayleigh number. At low values of $R a_{P}$ the Nusselt number values are near to 1 , the dominant heat transfer mechanism in this case is conduction. This changes with increasing the $R a_{P}$ and decreasing of $D a$, when convection becomes dominant while conduction is negligible. When values of $D a$ are high, the Brinkman viscous term in the momentum equation plays a significant role and reduces the overall heat transfer, which results in smaller values of $N u$. With decrease of $D a$, the influence of Brinkman viscous term becomes almost negligible $\left(D a<10^{-4}\right)$. In that case viscous effects become smaller and the inertial effect becomes significant due to high fluid velocity. For low values od Darcy number the model gives similar results as the classical Darcy model [17].

The presented results were obtained for the cases, where the thermal buoyancy force is the only acting force ( $L e=0$ and $N=0$ ). In addition, some results for double-diffusive natural convection, where $L e=10$ and $N=1$ are presented in table 2. In this case thermal and solutal buoyancy forces are aiding each other, which results in higher heat transfer and additional solute transfer through porous enclosure as can be observed from the values of Nusselt and Sherwood numbers in table 2.

In Fig. 3 the iso-surfaces for absolute value of $y$ velocity component are plotted. From the flow structure in the enclosure (velocity vectors) it can be examined, that the flow field is not far from being 2D. This is due to the fact that the flow field is driven by a temperature and concentration differences between two opposite walls, which causes large two-dimensional vortex in the $y$ plane. The 3D nature of the phenomena can be observed in the corners of the domain, as shown in Fig. 3. The extent of movement perpendicular to the plane of the main vortex is small, but it becomes more apparent in case of higher $R a_{p}$ and lower values of $D a$, in general.

\section{Conclusions}

Three-dimensional convective flow in a cube enclosure filled with saturated porous media was examined numerically using the boundary element method. 

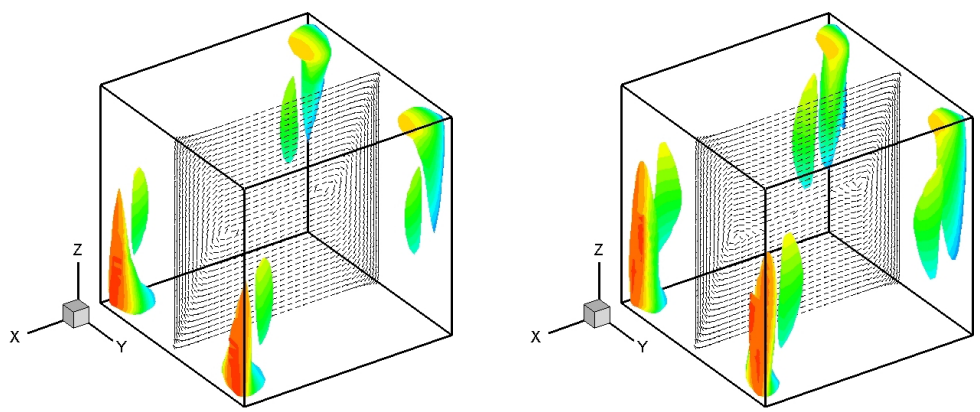

Figure 3: Iso-surfaces for $R a_{P}=500, D a=10^{-3}$ and absolute value of velocity component $\left|v_{y}\right|=3$ (left) and $R a_{P}=1000, D a=10^{-3}$, $\left|v_{y}\right|=7$ (right). Contours of temperature are displayed on the isosurfaces $(-0.5<T<-0.5)$. In addition, the velocity vectors on the plane $y=0.5$ are displayed.

The numerical algorithm is based on the combination of single domain and subdomain boundary element method, which are used to solve the velocityvorticity formulation of macroscopic Navier-Stokes equations. Some results of overall heat and solute transfer through enclosure are given in terms of Nusselt and Sherwood number values. It may be observed, that the flow regime as well as heat and solute transfer strongly depend on the values of the governing nondimensional parameters, e.g. Rayleigh, Darcy and Lewis number. 3D nature of the flow field is observed in the corners of the enclosure, although the fluid is moving predominantly in a single two-dimensional vortex.

\section{Acknowledgement}

One of the authors (J. Kramer) acknowledges the financial support to the research project Z2-2035 as provided by the Slovenian Research Agency ARRS.

\section{References}

[1] Stamps, D.W., Arpaci, V.S. \& Clark, J.A., Unsteady three-dimensional natural convection in a fluid-saturated porous medium. Journal of Fluid Mechanics, 213, pp. 377-396, 1990.

[2] Neto, H.L., Quaresma, J.N.N. \& Cotta, R.M., Natural convection in three-dimensional porous cavities: integral transform method. International Journal of Heat and Mass Transfer, 45, pp. 3013-3032, 2002.

[3] Neto, H.L., Quaresma, J.N.N. \& Cotta, R.M., Integral transform solution for natural convection in three-dimensional porous cavities: Aspect ratio effects. International Journal of Heat and Mass Transfer, 49, pp. 4687-4695, 2006. 
[4] Dawood, A.S. \& Burns, P.J., Steady three-dimensional convective heat transfer in a porous box via multigrid. Numerical heat transfer, Part A, 22, pp. 167-198, 1992.

[5] Sharma, R.V. \& Sharma, R.P., Non-Darcy effects on three-dimensional natural convection in a porous box. Annals of the Assembly for International Heat Transfer Conference 13, 0, pp. NCV-10, 2006.

[6] Wang, Q.W., Zeng, M., Huang, Z.P., Wang, G. \& Ozoe, H., Numerical investigation of natural convection in an inclined enclosure filled with porous medium under magnetic field. International Journal of Heat and Mass Transfer, 50, pp. 3684-3689, 2007.

[7] Sezai, I. \& Mohamad, A.A., Double diffusive convection in a cubic enclosure with opposing temperature and concentration gradient. Physics of Fluids, 12, pp. 2210-2223, 2000.

[8] Mohamad, A.A., Bennacer, R. \& Azaiez, J., Double diffusion natural convection in a rectangular enclosure filled with binary fluid saturated porous media the effect of lateral aspect ratio. Physics of fluids, 16, pp. 184-199, 2004.

[9] Ravnik, J., Škerget, L. \& Žunič, Z., Velocity-vorticity formulation for 3D natural convection in an inclined enclosure by BEM. Int J Heat Mass Transfer, 51, pp. 4517-4527, 2008.

[10] Ravnik, J., Škerget, L. \& Hriberšek, M., Analysis of three-dimensional natural convection of nanofluids by BEM. Eng Anal Bound Elem, 34, pp. 1018-1030, 2010.

[11] Popov, V., Power, H. \& Škerget, L., Domain Decomposition Techniques for Boundary Elements Application to Fluid Flow. WIT Press, Southampton, Boston, 2007.

[12] Bear, J., Dynamics of Fluids in Porous Media. Dover Publications, Inc., Mew York, 1972.

[13] Nield, D.A. \& Bejan, A., Convection in porous media (Third edition). Springer, 2006.

[14] Škerget, L., Hriberšek, M. \& Kuhn, G., Computational fluid dynamics by boundary-domain integral method. Int J Numer Meth Engng, 46, p. 1291.1311, 1999.

[15] Škerget, L., Hriberšek, M. \& Žunič, Z., Natural convection flows in complex cavities by BEM. Int J Num Meth Heat \& Fluid Fl, 13, pp. 720-735, 2003.

[16] Wrobel, L.C., The Boundary Element Method. John Willey \& Sons, LTD, 2002.

[17] Jecl, R., Škerget, L. \& Petrešin, E., Boundary domain integral method for transport phenomena in porous media. Int J Numer Meth Fluids, 35, p. 39.54, 2001. 